\title{
Lymph Node Blood Vessels: Exit Route for Systemic Dissemination of Cancer
}

\author{
Robert Skopec* \\ Researcher analyst, Dubnik, Slovakia, Europe

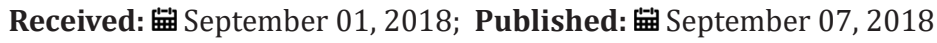 \\ *Corresponding author: Robert Skopec, Researcher analyst, Dubnik, Slovakia, Europe
}

\begin{abstract}
There are reports about the existence of meningeal lymphatic vessels in human and nonhuman primates (mormoset monkeys) and feasibility of noninvasively imaging and mapping them in vivo with high-resolution, clinical MRI. On T2-FLAIR and T1-weighted black-blood imaging, lymphatic vessels enhance with graduator, a gadolinium-based contrast agent with high propensity to extravasate across a permeable capillary endothelial barrier, but not with gadofosveset, a blood-pool contrast agent.
\end{abstract}

Keywords: Meningeal Lymphatic Vessels; The Cerebrospinal Fluid; The Dura Matter; The Superior Sagittal; Transverse Sinuses; Blood Brain Barrier; Chronic Inflammatory Response; Environmental Factors; Crc Risk

\section{Introduction}

The topography of these vessels, running alongside Dural venous sinuses, recapitulates the meningeal lymphatic system of rodents. In primates, meningeal lymphatics display a typical panel of lymphatic endothelial markers by immunochemistry. This discovery holds promise for better understanding the normal physiology of lymphatic drainage from the central nervous system and potential aberrations in neurological diseases.

\section{Material and Methods}

How does the brain rid itself of waste products? Other organs in the body achieve this via a system called the lymphatic system. A network of lymphatic vessels extends throughout the body in a pattern like that of blood vessels. Waste products from cells, plus bacteria, viruses and excess fluids drain out of the body's tissues into lymphatic vessels, which transfer them to bloodstream. Blood vessels then carry the waste products to the kidneys, which filter them out for secretion. Lymphatic vessels are also a highway for circulation of white blood cells, which fight infections, and are therefore an important part of the immune system. Unlike other organs, the brain does not contain lymphatic vessels. So how does it remove waste? Some of the brain's waste products enter via the fluid that bathes and protects the cerebrospinal fluid before being disposed of via the bloodstream. Recent studies in rodents have also shown the presence of lymphatic vessels inside the outer membrane surrounding the brain the dura matter. Some reports show that the dura matter of humans and mormoset monkeys contains lymphatic vessels too. Spotting lymphatic vessels is challenging because they resemble blood vessels, which are much more numerous. In addition, it was found a way to visualize the lymphatic vessels in the dura mater using brain magnetic resonance imaging and could confirm that lymphatic vessels are present in autopsy tissue using special staining methods. For magnetic resonance imaging, monkeys and human volunteers received an injection of a dye-like substance called gadolinium, which travels via the bloodstream to the brain. In the dura mater, gadolinium leaks out of blood vessels and collects inside lymphatic vessels, which show up as bright whiter areas on brain scans. To confirm that the white areas were lymphatic vessels, the experiment was repeated using a different dye that does not leak out of blood vessels. As expected, the signals observed in the previous brain scans did not appear. By visualizing the lymphatic system, this technique makes it possible to study how the brain removes waste products and circulates white blood cells, and to examine whether this process is impaired in aging or disease.

Some reports [1] described the existence of a network of true lymphatic vessels within the mammalian dura mater that runs alongside blood vessels, notably the superior saggital and transverse sinuses. The dural lymphatic vessels display typical immunohistochemical markers that identify lymphatic vessels elsewhere in the body. They provide an alternate conduit for drainage of immune cells and cerebrospinal fluid (CSF) from the brain, beyond previously described pathways of flow: via arachnoid granulations into the dural venous sinuses, and via cribriform plate 
into the ethmoid region. Although early reports, based on injections of India ink into the cisterna magna of the rat, suggested that the dural pathway accounts for only a minority of the drainage. The more recent studies, based on injections of fluorescent tracers and in vivo microscopy, indicate that the dural system may be substantially more important for drainage of macromolecules and immune cells than previously realized. Whether a similar network of dural lymphatics is present in primates is possible. A noninvasive visualization of the dural lymphatics is necessary to understanding their normal physiology and potential aberrations in neurological diseases. It was verified pathologically the existence of a dural lymphatic network in human and nonhuman primates (mormoset monkeys) and evaluated two magnetic resonance imaging (MRI) techniques that might enable its visualization in vivo.

First, the T2-weighted fluid-attenuation inversion recovery (T2-FLAIR) pulse sequence, which is the clinical standard for detecting lesions within the brain parenchyma and is highly sensitive to the presence of gadolinium-based contrast agents in the CSF. Second, black-blood imaging sequences, which are typically used for measurement of vascular wall thickness or detection of atherosclerotic plaque, are tuned to darken the contents of blood vessels (even when they contain a gadolinium-based contrast agent), but in the process the images may highlight vessels with other contents and flow properties. For comparison, is also required a postcontrast T1-weighted Magnetization Prepared Rapid Acquisition of Gradient Echoes (MPRAGE) MRI sequence, which is widely implemented for structural brain imaging and depicts avid enhancement of dura mater and blood vessels, but which would not be expected to discriminate lymphatic vessels. Cerebral blood vessels have a highly regulated blood-brain barrier, protecting the neutrophil from many contents of the circulating blood. Under physiological conditions, the blood-brain barrier prevents gadolinium-based chaltes in standard clinical use from passing into the Virchow-Robin perivascular spaces and parenchyma, so that these structures do not enhance on MRI. On the other hand, dural blood vessels lack a blood-menigeal barrier [1].

Darwinian selection process promotes spreading of the new long distant tumors. Cancer metastasis, the migration of cells from a primary tumor to form distant tumors in the organism, can be triggered by a chronic leakage of DNA within tumor cells, according to a team led by Weil Cornell Medicine and Memorial Sloan Kettering Cancer Center researchers. How metastasis occurs has been one of the central mysteries of cancer biology. The findings, published in Nature, appear to have partly solved this mystery. The authors traced the complex chain of events that results from chromosomal instability a widespread feature of cancer cells in which DNA is copied incorrectly every time these cells divide, resulting in daughter cells with unequal DNA content. Using models of breast and lung cancer, the investigators found that chromosomal instability leads to changes in the organisms that drive metastasis. They showed that chromosomal instability can cause a leakage of DNA from the nuclei of cancer cells, leading to a chronic inflammatory response within the cells. The cells essentially can hijack that response to enable themselves to spread to distant organs, said study lead author Dr. Samuel Bakhoum, a Holman research fellow at Weill Cornell Medicine and a senior resident in radiation oncology at Memorial Sloan Kettering Cancer Center. The discovery is principally a basic science advance but can also have long-range implications for cancer drug development. Metastasis cause 90 percent of cancer deaths, and this work opens new possibilities for therapeutically targeting it, said senior author Dr. Lewis Cantley, the Meyer Director of the Sandra and Edward Meyer Cancer Center and a professor of cancer biology at Weil Cornell Medicine. Prior studies have linked chromosomal instability to metastasis, although the reason for the link hasn't been clear. Starting hypothesis was that chromosomal instability generates a lot of genetically different tumor cells, and that a Darwinian selection process promotes the survival of the cells capable of spreading and forming distant tumors, Dr. Bakhoum said. When he injected chromosomally unstable tumor cells into mice, he indeed found that they were many times more likely to spread and form new tumors than tumor cells in which chromosomal instability was suppressed. That was true even though both sets of tumor cells started out genetically identical, with the same abnormal numbers of chromosomes, suggesting that chromosomal instability itself was a driver of metastasis. The researchers examined gene activity in these two sets of tumor cells. They found that those with high chromosomal instability had abnormally elevated activity stemming from more than 1,500 genes particularly in ones involved in inflammation and response of the immune system to viral infections.

These were cancer cells cultured in a dish, not in the presence of any immune cells, Dr. Bakhoum said.

Recent studies by other laboratories offered some clues: Chromosomes in unstable tumor cells can leak out of the cell nucleus where they normally reside. These mis-located chromosomes encapsulate themselves to form micronuclei in the fluid, or cytosol, in the main part of the cell outside of the main nucleus. However, micronuclei tend to rupture, releasing naked DNA into the cytosol. Cells interpret DNA in the cytosol as a sign of an infecting virus, which typically releases its DNA in the cytoplasm when it first attacks a cell. Human cells have evolved to fight this type of viral infection by sensing naked cytosolic DNA using a molecular machine called the cGAS-STING pathway. Once activated, this pathway triggers an inflammatory antiviral program. Lowering cGAS-STING levels reduced inflammation and prevented the ability of otherwise aggressive tumor cells to metastasize when injected into mice. In an ordinary cell, an antiviral response stimulated by DNA leakage from the nucleus would soon bring about the cell's death. The researchers found, however, that tumor cells have succeeded in suppressing the lethal elements of the cGAS-STING response [2]. At the same time, they use other parts of the response to enable themselves to detach from the tumor and become mobile within the organism. They start as in they were certain kinds of immune cells masking, which are normally activated by infection. 
In response, they move to the site of infection or injury in the body very quickly. By doing so, cancer cells engage in some form of lethal immune mimicry i. e. masking, and this Darwinian selection process metastasize into the social dynamics on the macroscopic level. The evidence is based on recent studies of metastatic tumor properties, that about half of human metastases originate and expand this way. Researchers are currently investigating strategies for blocking the process.

It might not be feasible to target chromosomal instability itself, since tumor cells are inherently prone to that. Chromosomally unstable tumor cells, with their cytosolic DNA, are basically full of their own poison. Undoing their ability to suppress normal and lethal antiviral response to cytosolic DNA would, in principle, kill these aggressive cancer cells swiftly, with minimal effects on other cells. The next step is to understand better how these cells alter the normal response and how it is possible to restore it [3]. Cancer cells often metastasize by hitching a ride on platelets.

\section{Results}

\section{An Alternate Route for Metastatic Cells}

Metastatic tumor cells are thought to reach distant organs by traveling through the blood circulation or the lymphatic system. Some studies of mouse models now suggest a hybrid route for tumor cell dissemination. Brown et al. used distinct methodologies to monitor the fate of tumor cells in lymph nodes. They found that tumor cells could invade local blood vessels within a node, exit the node by entering the blood circulation, then go on to colonize the lung. Probably this dissemination route occurs in cancer patients too. This answer could potentially change the way that affected lymph nodes are treated in cancer. During metastasis, malignant cells escape the primary tumor, intravasate lymphatic vessels, and reach draining sentinel lymph nodes before they colonize distant organs via the blood circulation. Although lymph node metastasis in cancer patients correlates with poor prognosis, it's a question how tumor cells enter the bloodstream via the lymph nodes of mice by microinfusing the cells into afferent lymphatic vessels. Tumor cells rapidly infiltrated the lymph node parenchyma, invaded blood vessels, and seeded lung metastases without involvement of the thoracic duct [4]. These results suggest that the lymph node blood vessels can serve as an exit route for systemic dissemination of cancer cells in experimental mouse models. We are convinced that this form of tumor cell spreading occurs also in cancer patients.

\section{Mysterious RNA Strands Avoid Cell Death}

Researchers from Case Western Reserve University School of Medicine have discovered how unusually long strands of RNA help colon cancer cells avoid death, allowing unregulated growth. Unlike other RNAs, the intriguing strands do not appear to encode proteins and are termed "long non-coding RNAs" or "lincRNAs." A new study showed some lincRNAs could be targeted by drug developers to halt colon cancer. In a new study published in Scientific Reports, researchers compared lincRNA levels inside tumor cells, to levels inside healthy colon cells. They found over 200 lincRNAs at significantly different levels inside the tumor cells as compared to normal cells. One, called lincDUSP, was overexpressed in 91 percent of the tumor samples. A few tumors had more than 15-times the normal amount of lincDUSP. The significant increase suggested this mysterious, a previously uncharacterized, RNA could be cancer-causing. To determine whether lincDUSP shows oncogenic activity in colon cancer, they decided to test the effects of depleting lincDUSP in patient-derived colon tumor cell lines. The researchers genetically modified colon cancer cells to deplete lincDUSP, and surprisingly, the cells began replicating at normal rates. They no longer had unrestricted growth associated with colon cancer tumor cells. Small molecules that inhibit lincDUSP, could have similar effects.

Above work demonstrates that not only protein-coding genes, but also non-coding genes contribute to colon cancer progression, says Ahmad Khalil, Ph.D., senior author, assistant professor of genetics and genome sciences at Case Western Reserve University School of Medicine, and member of the Case Comprehensive Cancer Center. LinsRNAs could be exploited as direct drug targets in this and other human diseases. Khalil's team discovered that depleting lincDUSP restored inherent cell death mechanisms. Colon cancer cells with low levels of lincDUSP became susceptible to cellular checkpoints that keep growth in check. They immediately committed cell suicide-apoptosis at the first sign of DNA damage. Depleting the single lincRNA also had widespread genetic effects. Khalil's team discovered that reducing lincDUSP levels affected expression of over 800 other genes. These results, combined with the team's experiments showing lincDUSP interacting with DNA, add to a growing body of evidence that lincRNAs are central to gene regulation. As such, they could represent an intriguing arena for drug developers. Not much is known about the role of long non-coding RNAs in colon cancer, says A. Khalil. Using new technologies that target RNA molecules instead of proteins, adds a new dimension to cancer therapies [5].

\section{Classical Immune Therapies Also Effective Against Cancer}

Tumors that develop at the transition of the stomach to the oesophagus, so called adenocarcinomas of the gastroesophageal transition (AEG), are still difficult to treat and the chances of recovery are still low. Researchers at the Comprehensive Cancer Center of Media Vienna and AKH Vienna have now been able to show that patients with non-metastatic AEGs have a better prognosis if their tumor cells produce the signal molecule PDL1. The study has now been published in the top journal Onco Immunology. In Austria, adenocarcinoma of the gastroesophageal transition is diagnosed in about 500 people every year. This type of tumor with by far the highest increase. Sebastian Schoppman, Department of Surgery (Head M. Gnant) of MedUni Vienna and AKH Vienna, Head of the Gastroesophageal Tumor Unit of CCC and the above study said: The increase is alarmingly high. According 
to study, one in 100 men in Europe will suffer from AEG in 2030. It is therefore particularly important to treat risk factors such as reflux early enough and to stop others, such as excessive alcohol consumption and smoking. The standard AEG therapy includes the surgical removal of the tumor, followed by chemotherapy and radiotherapy. Immunotherapy is also increasable becoming part of the treatment, which is why the complex process surrounding the immune response are of great interest to cancer researchers. The study team led by Dagmar Kollmann, Department of Surgery at MedUni Vienna and AKH Vienna, first author of the study and member of the CCC, investigated in retrospective work the patterns with which cancer and special defence cells PD-L1 and PD-L2 as well as associated receptor PD-1. They analyzed the tumor material of 168 patients and found that PD-L1 was detectable (expressed) in about 50 percent of cancer cells and TILs [6]. The PD- 1 receptor was formed in about 80 percent of the cells. They also found that the expression of PD-L1 in patients who had not been pre-treated with immunotherapy is an independent and powerful predictor of favorable disease progression while the presence of PD-1 is associated with poorer development and an advanced stage of the disease. Their study was able to identify a new biomarker that helps to manage given patients. In addition, the results suggest that all therapies directed against the $\mathrm{PD}-1$ receptor, i.e. classical immune therapies, are also effective in AEG. Loss of muscle mass represents a significant risk to esophageal cancer survival [7].

\section{Environmental Factors Explain a Significant Part of the Crc Risk}

Colorectal cancer (CRC) screening of the average risk population is only indicated according to age. Gemma Ibanez-Sanc et al. have elaborated a model to stratify the risk of CRC by incorporation of environmental data and single nucleotide polymorphism (SNP). The MCC-Spain case-control study included 1336 CRC cases and 2744 controls. Subjects were interviewed on lifestyle factors, family and medical history. Twenty one CRC susceptibility SNPs were genotyped. The environmental risk model included alcohol consumption, obesity, physical activity, red meat and vegetable consumption, and nonsteroidal anti-in amatory drug use, contributed to CRC with an average per factor OR of 1.36 (95\% Cl 1.27 to 1.45). Family history of CRC contributed an OR of 2.25 (95\% Cl 1.87 to 2.72), and each additional SNP contributed an OR of 1.07 $(95 \% \mathrm{Cl} 1.04$ to 1.10$)$. The risk of subjects with more than 25 risk alleles was $82 \%$ higher (OR $1.82, \mathrm{Cl} 1.11$ to 2.98 ) the subjects with less 19 alleles. This risk model, with an AUROC curve of $0.63(95 \%$ $\mathrm{Cl} 0.60$ to 0.66 ), could be useful be considered to encourage patients to achieve healthier lifestyle. Colorectal cancer screening by faecal occult blood testing has been demonstrated to reduce CRC incidence and mortality, as well as being a cost-effective strategy compared to no screening. Recent evidence of the balance of cancer screening has led to proposals for more personalized strategies based on individual cancer risk effectiveness of a screening strategy depends on the average cancer risk of the target population. Until today, the target population tested basically by age ( $>50$ years old), which has been called one-size-is-all strategy.

This strategy implies performing unnecessary screening tests in low-risk people leading to avoidable risks for patients and extra costs for the health care system. On the other hand, high-risk patients may receive non-invasive testing, which is a suboptimal screening technique in their case. A risk based CRC screening that included environmental risk factors, family history of CRC, and information derived from genetic susceptibility loci could improve not only the efficacy of the screening program but also the adherence of highrisk patients when properly informed of their personal risk. Several prediction models, either for CRC or advanced neoplasia, have been previously developed, all with limited discriminating ability. These studies have encompassed that traditional environmental risk factors for CRC including age, sex, family history of CRC, smoking, alcohol, Body Mass Index (BMI), acetylsalicyclic acid (ASA), physical activity, diet, some drugs (nonsteroidal anti-inflammatory drugs (NSAID), calcium and vitamins. Furthermore, with the identification of CRC-associated common single-nucleotide polyphormisms (SNPs), a few studies have added genetic-susceptibility information together with some of the clinical risk factors. Each common lowpenetrance allele is associated with a small increase in risk of CRC, but the combined test of multiple SNPs may achieve a higher degree of risk discrimination, which could be useful to stratify the population. In above study the researchers have developed a risk stratification model that combines environmental factors with family history and genetic susceptibility. They have interpreted the relative contribution of these factors and the utility of the model for risk stratification and public health intervention [6].

The above study assessed the potential utility of a risk prediction model for CRC that combines modifiable risk factors with family history of CRC and a genetic risk score based on 21 susceptibility SNPs. They have observed that modifiable risk factors have a stronger value for risk prediction than does genetic susceptibility. Though the added value of each SNP is small, the combination of 21 SNPs adds significantly to the power of the risk model. The study was large enough to confirm that established risk factors are associated with risk family history of CRC, high consumption of alcohol, obesity, lack of physical activity in leisure time, high intake of red meat, low intake of vegetables, and nonuse of NSAIDs/ASA. These risk factors were selected based on previous evidence reported in systematic reviews and metaanalyses. All were independent predictors of CRC in an average risk population, except for smoking, which was only significant in the univariate analysis. A recent meta-analysis on smoking has shown that the effect is small for CRC, with summary of smaller than 1.25, and larger for rectal than colon cancer. They also analyzed other covariant that have been associated with CRC (diabetes mellitus, inflammatory bowel disease, and diverticulitis), but they were not associated with CRC, perhaps because of the smaller number of a detected individuals. Nor was intake of vitamin D, calcium, or folic 
acid associated with CRC. Not was included statins in the model since there is controversy regarding these drugs and CRC risk.

Study confirms that the family history of CRC is the strongest single risk factor for CRC. They found a significant association between the CRC and family history and genetic factors, which highlights the importance of genetic susceptibility in CRC. Family history could also contribute to risk through shared lifestyle or environmental factors. Also, gene-environment interactions may play role in this type of cancer.

On average, each environmental factor increases risk by $35 \%$, while each risk allele only increases risk by $7 \%$. It implies that the change of one modifiable risk factor towards healthier lifestyle might set the effect of 4 risk alleles. Given the fact that environmental factors explain a significant part of the CRC risk, we believe it too important to give thought to incorporating clinical data to improve current screening and also encourage patients to achieve a healthier lifestyle [7].

\section{Acknowledgement}

The author gratefully acknowledge the assistance of Dr.Marta Ballova, Ing. Konrad Balla, Livuska Ballova, and Ing. Jozef Balla.

\section{References}

1. Absinta M, Ha SK, Nair G, Sati P, Luciano NJ, et al. (2017) Human and nonhuman primate meninges harbor lymphatic vessels that can be visualized noninvasively by MRI. ELife 3(6): e29738.

2. Akimov A (1992) Heuristic discussion of the problem of findinig longrange interactions. EGS-consepts Cise Vent preprint (7): 59.

3. Bakhoum SF, Nqo B, Lauqhney AM, Cavallo JA, Murphy CJ, et al. (2018) Chromosomal instability drives metastasis through a cytosolic DNA response. Nature 553(7689): 467-472.

4. Brown M, Assen FP, Leithner A, Abe J, Sixt M, et al. (2018) Lymph node blood vessels provide exit routes for metastatic tumor cell dissemination in mice. Science 359 (6382): 1408-1411.

5. Forrest ME, A Saiakhova, L Beard, AM Khalil (2018) Colon Cancer Upregulated Long Non-Coding RNA lincDUSP Regulates Cell Cycle genes and Potentiates Resistance to Apoptosis. Scientific Reports 8(1).

6. Ibanez Sanz, Alonso MH, Tardon A, Pollan M, Moreno V, et al. (2017) Risk Model for Colorectal Cancer in Spanish Population Using Environmental and Genetic Factors. Reslts from the MCC-Spain study. Scientific Reports $7(43263)$.

7. Kollmann D, Iqnatova D, Gnant M, Chanq YT, Jomrich G et al. (2018) PD-L1 expression is an independent predictor of favorable outcome in patients with localized esophageal adenocarcinoma. OncoImmunology 7(6): e1435226.

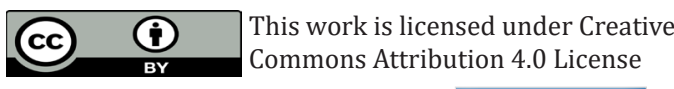

To Submit Your Article Click Here: $\quad$ Submit Article

DOI: $10.32474 /$ CTBM.2018.01.000104

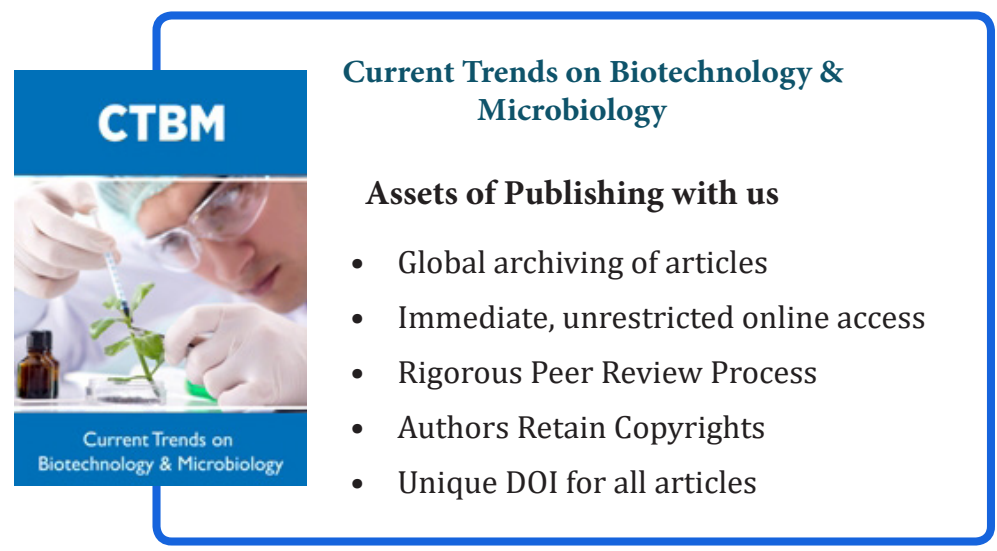

